\title{
Using Water with Oil Immersion Lens to Detect Malaria Parasite in Blood Film and Making a Comparison between Oil and Water Method
}

\author{
Hiba Siddig Ibrahim*, Shamsoun Khamis Kafi \\ Department of microbiology, Faculty of Medical Laboratory Science, The National Ribat University \\ *Corresponding author: hibasiddig55@gmail.com
}

Received April 21, 2014; Revised July 25, 2014; Accepted August 01, 2014

\begin{abstract}
Background: Oil immersion lens it is one of the microscope lenses that used in medical laboratory filed. It is used to magnify the smallest things and for detection of some important infectious diseases such as bacteria, parasite, anemia's and bone marrow films, but uses lens alone without oil makes the image imperfect and unclear, so to make a better image scientists were used oil with these lenses (immersion oils lens) to avoid bending of light [1,2]. Objectives: The main aim of this study is to be used distill water with oil immersion lens instead of synthetic oil to detect malaria parasite to low cost of using synthetic oil especially in faraway places due to difficulties of paying synthetic oil and also to be arrived to rural area, and so on to be used with other tests, beside avoiding using impurity oil due to the comment of D/ Fahad Awad (National Coordinator for Malaria Program), against using sesame oil especially in some laboratories at Al Gedaraf State, see Aray Alaam newspaper 5th December, 2013. Methodology: In this study a total of 200 subjects were included. A 3 drops from capillary blood samples were collected in clean dry dust free slides after disinfectant the 3ed or 4th finger for adult and the big toe hand or foot for child with $70 \%$ alcohol to make a thick blood films, or Aliquots of $2.5 \mathrm{ml}$ of venous blood were collected by venous puncture after disinfectant the site of collection and the collected blood was drawn into EDITA containers to make thick blood films as above. The films were lets to dry by air, staining with Giemsa stain for 10 minute and then examined for the first times with DW with objective lens and for the second times with synthetic oil. Results: There were no differences between using oil or distill water (DW) for detection of malaria parasite by both techniques (100\%), unless the high quality of oil image (100\%), when comparing with distill water (DW) image (90\%), due to variation in numerical aperture (NA) between both techniques. Conclusion: Based on the results of this study we can used distill water (DW) but with caution to detect malaria parasite by $90 \%$ when compared with oil $100 \%$ in case of poverty areas to low cost of using synthetic oil, and so on the image quality can arrives (99.5\%) if do it in proper way.
\end{abstract}

Keywords: distill water (DW), objective lens, bending, numerical aperture (NA)

Cite This Article: Hiba Siddig Ibrahim, and Shamsoun Khamis Kafi, "Using Water with Oil Immersion Lens to Detect Malaria Parasite in Blood Film and Making a Comparison between Oil and Water Method.” American Journal of Microbiological Research, vol. 2, no. 4 (2014): 122-124. doi: 10.12691/ajmr-2-4-4.

\section{Introduction}

Oil immersion lens it is one of the microscope lenses with wide uses in medical laboratory filed. It used to magnify the smallest things and for detection of some important infectious diseases also such as bacteria, parasite, and anemia's and bone marrow films but uses lens alone without oil make the image imperfect and unclear, so to make a better image scientists were used oil with these lenses (immersion oils lens) to avoid bending of light $[1,2]$. The upper limit of the resolving power of light microscopes is slightly above $1000 \mathrm{X}$. Objectives of 90 to $100 \mathrm{X}$, when coupled with a $10 \mathrm{X}$ eyepiece, approach that upper limit. Even in the range of 900 to $1000 \mathrm{X}$, a clear image is only possible if every bit of available light is directed through the microscope optics to the viewer's eye. Immersion oils play an essential role in maximizing the amount of light producing the image the viewer sees [1]. This study is conducted to throw light on using DW with oil objective lens to detect malaria parasite in Sudan because every year we have a challenge in the fighting Malaria Program, due to different reasons such as improper uses of nets, drugs, sanitary especially in winter and autumn, even in lab technician. Although the different trails of both ministry of health and lab coordinator, that started from the simplest ways to fight mosquitoes to training lab technician with self-education of people. The current ways to diagnosis malaria parasites were not sufficient and it needs to be up dated especially with the appearance of other species of malaria for example Plasmodium falciparum was the only species found in El manshia, in Ed dekheinat P. falciparum, Plasmodium 
ovale and Plasmodium vivax constituted 84.9, 8.2 and $6.9 \%$ of the cases, respectively. Plasmodium ovale appears to have recently spread into Khartoum since it has not previously been reported there [3].

\section{Material \&Method}

This is a prospective experimental study which was conducted in The National Ribat University, from 1/2/2014-31/5/2014.

In this study a total of 200 subjects were included. 3 drops from capillary blood sample were collected in clean dry dust free slide after disinfectant the 3ed or 4th finger for adult and the big toe hand or foot for child with $70 \%$ alcohol to make thick blood films, or Aliquots of $2.5 \mathrm{ml}$ of venous blood were collected by venous puncture after disinfectant the site of collection and the collected blood was drawn into EDITA containers to make thick blood films as above. The films were let to dry, staining with Giemsa stain ( Concentrated Giemsa stain will be given from The Red Crescent) for 10 minute and examined for the first times with distill water (DW) by putting one drops of DW between films and oil objective lens and for the second times by putting oil between both films and oil objective lens.

The principle of objective lens depend on using the beam of light passes from air into glass it is bent and when it passes back from glass to air it is bent back again to its original direction, and this affect with high power lenses due to limit the amount of light which can enter the lens, affect the numerical aperture (NA) of the lens and consequently its resolving power. To avoid bending effect we can put oil to replace the air between the specimen and the lens which has the same refraction index as glass and this make the light passes in a straight line from glass through the oil and back to glass as though it were passing through glass all way.

\section{Results}

Malaria parasite can be detected by using both DW and synthetic oil as in Table 1

Table 1. Detection of malaria parasite by both synthetic oil and DW under microscope

\begin{tabular}{|c|c|c|}
\hline \multirow{2}{*}{ Method } & \multicolumn{2}{|c|}{ Ringe stage of malaria parasite under microscope } \\
\cline { 2 - 3 } & Nucleus & Cytoplasm \\
\hline Synthetic oil & Red & Blue \\
\hline DW & Red & Blue \\
\hline
\end{tabular}

There was no difference between using synthetic oil and DW for detection of malaria parasite as in Table 2.

Table 2. Comparisong between positive synthetic oil and DW

\begin{tabular}{|c|c|c|c|}
\hline \multirow{2}{*}{ Method } & \multicolumn{3}{|c|}{ Positive malaria parasite among studied group } \\
\cline { 2 - 4 } & Total examined & Frequency & Percentage \\
\hline Synthetic oil & 200 & 185 & $92.5 \%$ \\
\hline DW & 200 & 185 & $92.5 \%$ \\
\hline Total & 400 & 370 & $92.5 \%$ \\
\hline
\end{tabular}

There was a little difference between image quality of both synthetic oil and DW, without any difference between both techniques for detection of malaria parasite as in Table 3.
Table 3. Comparisons between image quality, and percentage of detections of malaria parasite by both techniques oil and DW

\begin{tabular}{|c|c|c|}
\hline Method & Image quality & Percentage of detection malaria parasite \\
\hline Synthetic oil & $100 \%$ & $100 \%$ \\
\hline DW & $90 \%$ & $100 \%$ \\
\hline
\end{tabular}

\section{Discussion}

According to this study of using distill water (DW) to detect malaria parasite with objective lens, it gives good results. In DW examination, 185 (92.5\%) were found to be positive for malaria parasite, while 15 (7.5\%) were found to be negative for malaria parasite. However the results were the same when compared by using synthetic oil, 185 (92.5\%) were found to be positive for malaria parasite, while 15 (7.5\%) were found to be negative for malaria parasite by oil, also there was no difference between both techniques to detect malaria parasite (100\%).

Actually the quality of image is about $90 \%$ and could be arrived $99.5 \%$ if the stain techniques was doing in the proper ways with addition to a good quality of stain itself, when compared with oil $100 \%$, due to different numerical aperture (NA) between both water (1.33) and oil (1.52), so on to fix this problems and to gives a better image we need to change NA of objective lens to 1.33 to fit with water or to made other lens to be used with water by the same principle of objective lens $[1,2,4,5]$. Furthermore the current ways to diagnosis malaria parasites were not sufficient due to in some cases we can found the symptoms of malaria but under the microscope we cannot found the parasite or it is below the limited number to be positive, beside this we actually known the advantages and disadvantages of rapid test, even doctor cannot differentiate between old remaining infections and new one, and those infected people noticed to be slightly resistant to drugs, may be due to new species or subspecies or even due to patients itself cannot take the doses of drug in the right time or not completed the course of treatment, in labs sometimes we cannot found the synthetic oil because it is very expensive especially for poor country and difficulty to be arrived far way places, beside improper concentrated Giemsa stain, and old malaria detection techniques.

\section{Conclusion}

Based on the results of this study we can use DW but with caution to detect malaria parasite by $90 \%$ when compared with synthetic oil 100\% image.

\section{Recommendations}

According to this study we can use water instead of oil but with caution due to water may be lead to erosion of lens body, beside this we need to alternate NA of objective lens to be 1.33 to fit with water NA to give better image or to add a substance that can rise up the NA of water.

Other oils with refractive index similar or close similar to 1.52 can be used, but with caution because we do not know if itcan effect on lens or not, and temperature should 
not be below $25^{\circ} \mathrm{C}$ to avoid condensation of oil, so on, at the end we need to do many research on these ways to found the more suitable one. I also recommended finding suitable but more accurate techniques to help in diagnosis of malaria with continuous researching on it.

\section{Acknowledgments}

I would like to acknowledge the inspirational instruction and guidance of Dr. Shamsoun KhamisKafi, also I would like to thank the family of the College of Medical Laboratory Science, The National Ribat University, and the patients without whom this research would have never seen the light.

\section{References}

[1] Cheesbrough M (2004). Microscope. In: District Laboratory Practice in Tropical Countries. Part 1, Low price Egyptian edition 2004. The Anglo-Egyptian Bookshop \& Cambridge University Press, pp: 110

[2] Teacher Resources. What is immersion oil used for and what is the proper technique for using it? (2013).

http://whatisimmersionoilusedforandwhatisthepropertechniqueforu singit.htm.

[3] El Sayed BB, Arnot DE, Mukhtar MM (2000).A study of the urban malaria transmission problem in Khartoum. Acta Trop. 2000 Mar 25; 75 (2): 163-71. http://www.ncbi.nlm.nih.gov/pubmed/10708656

[4] Che J (2005). Index of Refraction of Glass, Crown. http://hypertextbook.com/facts/2005/Justin Che.shtml

[5] Solliday J (2007). History of Oil Immersion Lenses. http://www.smecc.org/history_of_oil_immersion_lenses.htm 